\title{
KIKUCHI -FUJIMOTO DISEASE- A COMPREHENSIVE REVIEW
}

\author{
MD ROBED AMIN
}

\section{Introduction:}

Kikuchi- Fujimoto (KFD) disease was first reported initially in Japan in 1972. KFD was reported almost simultaneously by Kikuchi ${ }^{1}$ and by Fujimoto ${ }^{2}$ and associates as a lymphadenitis with focal proliferation of reticular cells accompanied by numerous histiocytes and extensive nuclear debris ${ }^{3}$.

The disease is an extremely rare disease known to have a worldwide distribution with a higher prevalence among Japanese and other Asiatic individuals ${ }^{4}$. Although first descriptions were in people of Asian origin, the disease has now been reported in individuals of all races. KFD is an enigmatic, benign and self-limited syndrome characterized by regional lymphadenopathy with tenderness, predominantly in the cervical region, usually accompanied by mild fever and night sweats ${ }^{4,5}$. Clinicians are unfamiliar with this entity. Here is a comprehensive review of KFD with special emphasis on the clinicopathologic significance of this condition.

\section{Literature Review:}

Relevant articles published in English language medical journals from 1972 (From first reported KFD) to the present day through a MEDLINE search using the keywords "kikuchi" "Fujimoto" and "histiocytic necrotizing lymphadenitis." In addition to original, review articles, correspondence, letters , editorials outstanding case reports and series are also included

\section{Epidemiology:}

Although uncommon, Kikuchi disease has been reported throughout the world and in all races. Most cases have been reported from East Asia, with fewer cases from Europe and North America. ${ }^{6-12}$.Outside of Asia, it is possible that Kikuchi disease has been underdiagnosed and therefore underreported. Dorfman and Berry reported 108 cases, including 68 in the United States; 63\% of the 108 patients were white. Cases reported by Pileri et al ${ }^{13}$ included 23 from the former West Germany and individual cases from Iran,
Italy, South Korea, and Spain and many other countries. India and Bangladesh has also reported many case reports on variable clinical presentation of KFD 9,14

The initial studies of Kikuchi disease reported women were affected more often than men by a ratio of approximately 3:1. However, more recent studies have shown a smaller female preponderance, with a ratio closer to $1.25: 1^{15-17}$.. Kikuchi disease occurs in a wide age range of patients (ie, 2-75 y), but it typically affects young adults (mean age, 20-30) 17

\section{Aetiology and Pathophysiology:}

The cause of Kikuchi disease is unknown, although infectious and autoimmune etiologies have been proposed. ${ }^{18}$ Among the infectious ages including viruses, many has been described in literatures due to presence of serological positivity in clinical courses. Yersinia enterocolitica and Toxoplasma gondii as possible causative agents of KFD was first reported due to presence of serological evidence but later found the histology to be different ${ }^{19}$. Among the viruses Epstein barr virus and Cytomegalovirus were the initial agents thought to be responsible with similar positive serology but later PCR studies were inconclusive. ${ }^{20,21}$ Herpes virus 6 and 8 have been also scrutinized to see the association. HTLV, Parvovirus B19, Varicella zoster, Hepatitis B were also searched for identifying aetiology but later found to have no association with core disease process ${ }^{22-}$ 26. A viral infection is still a possibility by characteristics clinical manifestations, as described by Unger and colleagues ${ }^{6}$ (upper respiratory prodrome, atypical lymphocytosis, and lack of response to antibiotic therapy.

Histologic, ultrastructural, and immunohistochemical findings might support an immunological reaction, perhaps to multiple organisms. It is possible that KFD might represent an exaggerated T cell-mediated immune response in genetically susceptible people to a variety of nonspecific stimuli. The incidence of

Address of Correspondence: Dr Md Robed Amin, Associate Professor of Medicine, Dhaka Medical College, Apt-C-2, House-76, Block-F, Road-5, Bonani, Dhaka. Email-robedamin@yahoo.com 
DPA $1{ }^{*} 01$ and DPB $1{ }^{*} 0202$ alleles of HLA antigen LAHLA is significantly higher in patients with KFD than in healthy control subjects. These genes are extremely rare or absent among Caucasians but relatively common among Asiatic people ( Korean, 9.9\%; and Japanese, 4.5\%). This might provide explanation about the mentioned epidemiologic pattern. ${ }^{28}$ Because tubular reticular structures in the cytoplasm of stimulated lymphocytes and histiocytes has been seen in patients with KFD, which has also been described in endothelial cells and lymphocytes of patients with systemic lupus erythematosus

(SLE) and other autoimmune disorders, Imamura and coworkers 27 hypothesized that KFD might reflect a selflimited SLE-like autoimmune condition induced by virus infected transformed lymphocytes. But the repeated test for ANA or other specific serologolical test for SLE was found negative in most of the classical cases of KFD which rules against the autoimmune process. Still the association between KFD and SLE has been reported with a frequency probably greater than that expected by chance alone.

The immunophenotype of KFD typically consists of a predominance of T-cells, with very few B cells. There is an abundance of CD8+ T-cells over CD4+. ${ }^{29}$ The histiocytes express histiocyte-as-sociated antigens such as lysozyme, myeloper-oxidase (MPO) and CD68. Finally, striking plas-macytoid monocytes are also positive for CD68 but not for MPO.1 Tcell- restricted intracellular antigen-1 (TIA-1) cytotoxic granules were detected within the cytoplasm of apoptotic bodies in KFD necrotizing lesions. ${ }^{30}$ In double stainings, TIA$1+$ lymphocytes were found to be CD8+ rather than $\mathrm{CD}+^{31}$. Ohshima and coworkers ${ }^{32}$ studied perforin and Fas pathways as CD8+ T-cell cytotoxic mechanisms that could induce apoptosis in target cells in patients with KFD. According to their observations, proliferating CD8+ $\mathrm{T}$ cells might act as "killers" and victims" in the apoptotic process via the Fas and perforin pathways.

The plasmacytoid monocytes or plasmacytoid T cellshave been reported to be a hallmark histopathologic finding of KFD. These cells would have a role in the pathogenesis of KFD via their migration from bone marrow to affected lymph nodes, where they might produce large amounts of type I interferon, thus promoting a T-helper $1 \mathrm{~T}$-cell response and the aforementioned cytotoxic immune reaction. ${ }^{33,34}$. Monocyte and macrophage lineage cells have been proposed as enhancers of the apoptotic event. Abe and coworkers ${ }^{35}$ recently demonstrated the presence of receptor-binding cancer antigen expressed on SISo cells (RCAS1) in the macrophages from lymph nodes of patients with KFD.

The authors suggested that the high presence of RCAS1 on macrophages might contribute to the histiocyte-dependent CD8+ T-cell death. ${ }^{35}$

Serum concentrations of some inflammation mediators such as interferon (IFN)-ã, FasL, and interleukin- 6 have been reported to be increased during the acute phase of KFD, returning to normal levels during the convalescent phase, thus raising the possibility that these cytokines could have a role in the pathogenesis of this condition. ${ }^{36}$. There has been report of cases where other mediators like interleukin-18, monokine induced by IFN-ã, and Cys$\mathrm{X}$-Cys chemokine IFN-ã-inducible protein-10 has been identified in histiocyte and lymphocyte. In few case report the complement level has been found reduced (especially $\mathrm{C} 4$ ) which is also commonly seen in patient with SLE. Low C4 levels in patients perhaps contributed to impaired clearance of immune complexes and may have predisposed to KFD.

\section{Pathology:}

KFD is diagnosed on the basis of an excisional biopsy of affected lymph nodes. Kuo TT5 proposed three histologic phases of KFD: proliferative, necrotizing and xanthomatous. The initial proliferative phase features an expanded paracortex with increases in various histiocytes, plasmacytoid monocytes, which are admixed with lymphocytes and nuclear debris. The necrotizing phase is characterized by presence of necrosis of any degree. If foamy histiocytes predominate in the lesions, the case is categorized as being in the xanthomatous phase despite the presence or absence of necrosis. These three histologic types of KFD could represent different evolving stages of the disease, however, this speculation has not been confirmed because of a lack of studies with sequential biopsies. Histological features Involved lymph nodes which are partially effaced architecture by paracortical nodules of apoptotic necrosis with abundant karyorrhectic debris and large numbers of histiocytes (fig. 1). The plasmacytoid monocytes tend to cluster, particularly at the margins of the necrotic foci. Admixed are many small lymphocytes and immunoblasts. Reactive immunoblastic component in some cases may be mistaken for lymphoma. Neutrophils and eosinophils are absent and plasma cells are scarce or absent. ${ }^{37,38}$ Thrombosed vessels may be present in regions, peripheral to necrosis. Reactive lymphoid follicles are 
observed in most cases. The karyorrhectic process can extend beyond the nodal capsule into perinodal tissue. ${ }^{37}$ Biopsies of skin lesions from patients with KFD revealed dermal infiltration by apoptotic plasmacytoid monocytes.

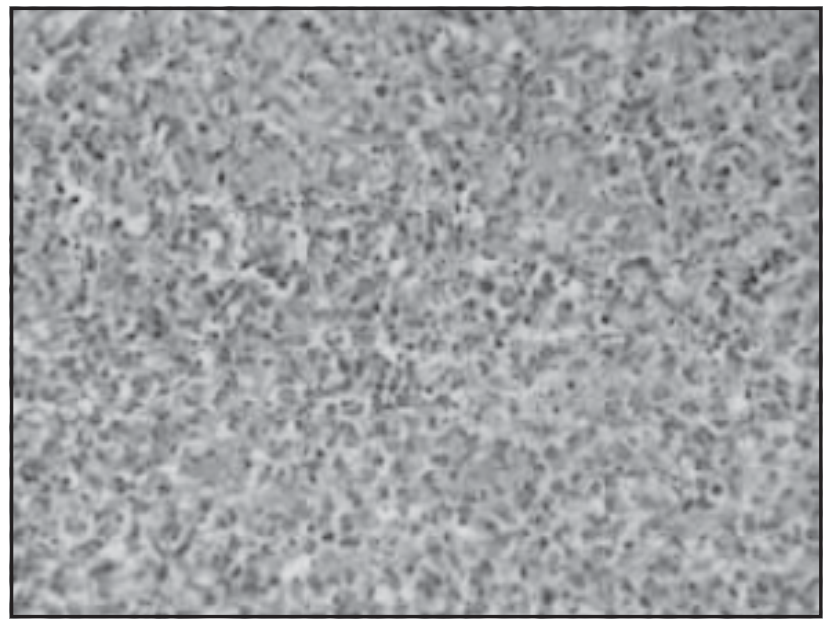

Fig 1: Lymph node biopsy material showing apoptotic necrosis with abundant karyorrhectic debris and large numbers of histiocytes

The cytological smears demonstrate characteristic intra and extracellular apoptotic nuclear debris with admixed crescentic macrophages (fig. 2) on a reactive lymphoid background. ${ }^{39}$ In some cells, nuclei may be ragged, dense and pyknotic as the apoptosis progresses. Free nuclear fragmentation may be present in the background as small, variably sized dark staining karyorrhectic globule. Neutrophils are notably absent and large numbers of interspersed small mature lymphocytes and some larger transformed lymphocytes are also present.

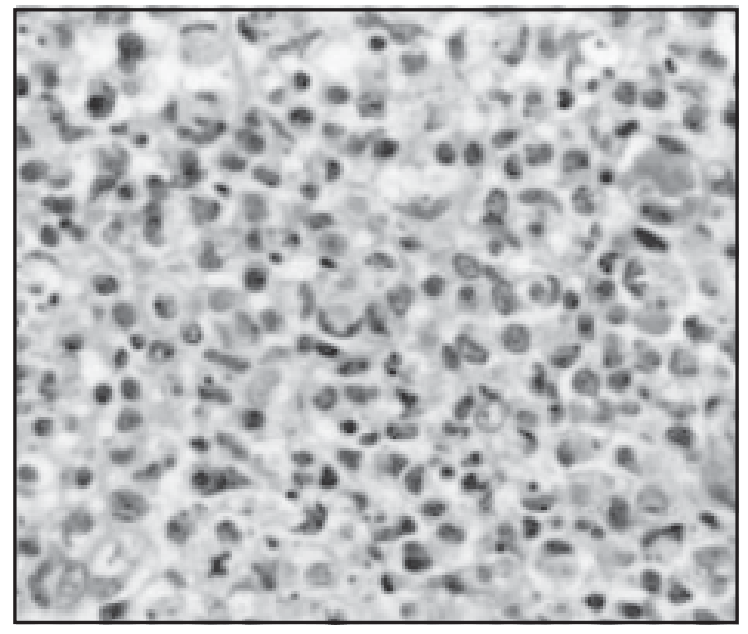

Fig 2: Karyorrhectic foci with large numbers of histiocytes, including crescentic histiocytes (arrow), and some lymphoid cells

\section{Clinical Manifestations: History:}

Kikuchi disease most frequently manifests as a relatively acute onset of cervical adenopathy associated with fever and a flulike prodrome. Cervical nodes are affected in about $80 \%$ of cases.Posterior cervical nodes are frequently involved $(65-70 \%$ of cases). Lymphadenopathy is isolated to a single location in $83 \%$ of cases, but multiple chains may be involved. Lymph node size ranges from 0.5 to $4 \mathrm{~cm}$ (93.4\%), and occasionally, lymph nodes are larger than $6 \mathrm{~cm}$. Painful lymphadenopathy is seen in up to $59 \%$ of patients. Generalized lymphadenopathy has been reported in $1 \%$ to $22 \%$ of cases. ${ }^{40,41,42.43 .44}$.Cases of generalized adenopathy involving axillary, inguinal, and mesenteric nodes are unusual.

A flulike prodrome with fever is present in $50 \%$ of cases. The less common symptoms are Headache, nausea, vomiting, malaise, fatigue, weight loss, arthralgias, myalgias, night sweats rash (up to $30 \%$ ), thoracic/abdominal pain

\section{Physical Sign:}

Lymphadenopathy is the hallmark sign in KFD.isolated to 1 location in $83 \%$ of patients, although multiple nodal chains may be involved.Cervical nodes are affected in $80 \%$ of patients; Less commonly affected nodes include those in axillary, mediastinal, celiac, abdominal, and inguinal locations. The nodes are usually described as painless or mildly tender. The nodes tend to be 2$3 \mathrm{~cm}$ in diameter, although masses of multiple nodes may reach $6 \mathrm{~cm}$. The nodes are usually firm and mobile, but they are not fluctuant or draining.

Extranodal findings are Skin ${ }^{45,46}$ - The incidence of skin involvement varies from 5-30\%. Findings are varied and nonspecific and include maculopapular lesions, morbilliform rash, nodules, urticaria, and malar rash, which may resemble that of SLE. Skin lesions resolve in a few weeks to months. Hepatosplenomegaly is not uncommon. Neurologic involvement is rare but has included conditions such as aseptic meningitis, acute cerebellar ataxia, and encephalitis ${ }^{47}$ Patients with aseptic meningitis may report headache, but they do not exhibit nuchal rigidity or positive Kernig or Brudzinski signs.

Uncommon features and associated clinical condition:

KFD also has been reported as a cause of prolonged fever of unknown origin. ${ }^{48}$. SLE has developed in some patients thought to have true KFD. The association with SLE is such intensive in few studies that KFD was thought to be an incomplete form of an 
autoimmune condition. The patients, however, may have had SLE from the beginning, taking into account the histopathologic consideration. ${ }^{49,50,51}$ The diagnosis of KFD can precede, postdate, or coincide with the diagnosis of SLE. ${ }^{50}$. Six cases of pre-SLE or post-SLE necrotizing lymphadenitis were found to be true KFD although authors concluded that the association of SLE and KFD is not linked as per the histological diagnosis concerned. ${ }^{51}$ Antiphospholipid syndrome, polymyositis, systemic juvenile idiopathic arthritis, bilateral uveitis, arthritis, cutaneous necrotizing vasculitis, and pulmonary hemorrhage have been linked to KFD which may also be explained by overlap syndrome with SLE. ${ }^{52-58}$. But there are report of severe KFD leading to fatality which were found to have related with severe autoimmune disorder. It has been reported rarely in HIV-positive patients 59 and in association with the human T-cell leukemia/lymphoma (lymphotropic) virus type 1 (HTLV-1) presented with recurrent asceptic meningitis.

Some cases of KFD have been associated with the hemophagocytic syndrome. ${ }^{60,61}$ and which also have severity in presentation. There have been other reports of unusual features of KFD, including Dysphagia ${ }^{14}$, parotid gland involvement ${ }^{62}$, thyroiditis, 63 carcinoma, ${ }^{64}$ and diffuse large B-cell lymphoma ${ }^{65}$ Skin lesions in KFD seem to be multivariant and nonspecific. Varieties of dermatological patterns including papules, nodules; erythematous, induration, erythematous lesions; erythema multiforme; and erythematous maculopapular eruptions, psoriaform eruption mainly affecting the face and upper body. ${ }^{66,67}$ Involvement of extranodal sites by KFD is uncommon, but skin and bone marrow involvement and liver dysfunction have been reported. 68

\section{Lab diagnosis:}

In patients with Kikuchi disease, diagnostic laboratory and radiologic test findings are nonspecific. Complete blood cell ( $\mathrm{CBC}$ ) count revealed Mild granulocytopenia is observed in $20-50 \%$ of patients. Leukocytosis is present in $2-5 \%$ of patients. Atypical lymphocytes are observed in $25 \%$ of patients.Erythrocyte sedimentation rate (ESR) and C-reactive protein (CRP) levels may be elevated. Elevated LDH levels suggest hepatic involvement.Results from autoimmune antibody studies, including LE preparation and RF and ANA studies, are generally negative. These findings may help the clinician distinguish Kikuchi disease from SLE. There are cases where KFD was diagnosed by histopathology and serology of SLE is positive which is perhaps indicative of common association with this autoimmune disorder.

\section{Imaging Study:}

Chest radiography are generally unremarkable in KFD disease and is recommended in the evaluation of cervical adenopathy to look for evidence of tuberculosis or malignancy. Ultrasonography 69 showed enlarged nodes may be either homogeneous or heterogeneous with hyperechoic rims. Computed tomography (CT) scanning and magnetic resonance imaging (MRI) usually revealed enlargement of lymph nodes in affected areas is noted. Postcontrast enhancement may be observed. A large series on CT scan by Kwon et al.${ }^{70}$ found homogeneous lymph node enlargement in $83.3 \%$ of the patients, perinodal infiltration in $81.3 \%$, and prominent areas of low attenuation suggestive of focal necrosis in $16.7 \%$.

\section{Tissue Diagnosis:}

Although results of fine-needle aspiration (FNA) may be suggestive, ${ }^{70,71}$-the diagnosis of Kikuchi disease is confirmed only by excisional lymph node biopsy. FNA findings are most often nonspecific, Although some authors believe that the diagnosis can be confirmed when supported by typical clinical findings. Most authors recommend confirmation by excisional biopsy. In a retrospective study of 44 patients, FNA had an overall accuracy of $56.75 \%$ in diagnosing Kikuchi disease. ${ }^{70}$-Characteristic cytologic findings in Kikuchi disease include crescentic histiocytes, plasmacytoid monocytes, and extracellular debris. Excisional lymph node biopsy revealed Paracortical necrosis which may be patchy or confluent. Crescent shaped nuclei in histocyte is characteristics. Lymphocytes, plasmacytoid monocytes, macrophages, and immunoblasts (predominantly $\mathrm{T}$ cells) are abundant at lesion site while karyorrhexis are occasionally seen. Neutrophils, granulomas, plasma cells are virtually absent.

\section{Treatment:}

\section{Supportive care:}

There is no specific treatment for patients with KFD. The disease is chacteristically self limited. Only symptomatic treatment measures to relieve distressing local and systemic complaints should be used. Nonsteroidal anti-inflammatory drugs (NSAIDs) may be used to alleviate lymph node tenderness and fever. Analgesics, antipyretics, and rest are the principal modality of treatment.

\section{Steroids:}

The use of corticosteroids, such as prednisone, has been recommended in severe extranodal or generalized Kikuchi disease. ${ }^{73,74}$ Indications for corticosteroid use include the following: 


$\begin{array}{ll}\text { o } & \text { Neurologic involvement } \\ \text { o } & \text { Aseptic meningitis } \\ \text {. } & \text { Cerebellar ataxia } \\ \text { Hepatic involvement - Elevated LDH level } \\ \quad \text { Severe lupuslike syndrome - Positive ANA } \\ \text { titers }\end{array}$

Jang and colleagues recommended expanding the indications for corticosteroid use to less severe disease. ${ }^{74}$-They administered prednisone when patients had prolonged fever and annoying symptoms lasting more than 2 weeks despite NSAID therapy, as well as for recurrent disease and for patients who desired a faster return to work.

\section{Other new agents:}

Takada and coworkers 111 recently reported a case of KFD that dramatically resolved with oral minocycline treatment, suggesting that the causative agent of KFD might be especially sensitive to this antibiotic. Immunosuppressants have been recommended as an adjunct to corticosteroids in severe, life-threatening disease.

Intravenous immunoglobulin (IVIG) was chosen in few cases of refractory KFD cases because, compared with cyclophosphamide, anti-tumour necrosis factor (TNF) antibodies (e.g. infliximab) or soluble TNF receptor (etanercept), it incurred least risk of adverse effects. ${ }^{75}$ The specific therapeutic effect of IVIG in a patient presenting solely with Kikuchi's disease was described in three case reports. ${ }^{75}$ Two case reports describe a therapeutic response to combined treatment with IVIG and corticosteroids in children with haemophagocytic syndrome and concurrent Kikuchi's lymphadenitis 76,77. Antiidiotype antibodies against autoantibodies and competition with endogenous autoantibodies for binding of $\mathrm{Fc}_{-}$ receptors or complement components may be important in autoimmune diseases. In view of its pluripotent effects, IVIG therefore represents a suitable candidate for empirical treatment of idiopathic inflammatory conditions such as Kikuchi's disease.

\section{Conclusion:}

KFD is rare, self-limiting disease with spontaneous remission of unknown etiology. The diagnosis of KFD should be consider in any nodal biopsy (especially cervical) showing fragmentation, necrosis, and karyorrhexis, especially in young people. Its diagnosis is important to prevent misdiagnosis of tuberculosis, lymphoma or carcinoma. The viral etiology has always on the card although there is no ample evidence to support the viral theory. The association with SLE should be also an important consideration as severe disease may respond well with steroids and even with IVIG. Awareness of this disorder by clinicians might help prevent misdiagnosis and inappropriate treatment.

\section{References:}

1. Kikuchi M. Lymphadenitis showing focal reticulum cell hyperplasia with nuclear debris and phagocytes: a clinicopathological study. Acta Hematol Jpn.1972; 35:379-80

2. Fujimoto Y, Kozima Y, Yamaguchi K. Cervical subacute necrotizing lymphadenitis: a new clinicopathologic entity. Naika. 1972; 20:920-27.

3. Dorfman RF. Histiocytic necrotizing lymphadenitis of Kikuchi and Fujimoto [editorial]. Arch Pathol Lab Med.1987;111:1026-1029.

4. Bosch X, Guilabert A, Miquel R, Campo E: Enigmatic Kikuchi-Fujimoto disease: a comprehensive review. Am J Clin Pathol 2004,122:141-152.

5. Xavier Bosch, Antonio Guilabert. Kikuchi-Fujimoto disease: Review. Orphanet Journal of Rare Diseases 2006, $1: 18$

6. Unger PD, Rappaport KM, Strauchen JA. Necrotizing lymphadenitis (Kikuchi's disease): report of four cases of an unusual pseudo-lymphomatous lesion and immunologic marker studies. Arch Pathol Lab Med. 1987; 111: 1031-1034.

7. Ahmad $M$, Khan $A B$, Iqbal J. Histiocytic necrotizing lymphadenitis: a clinicopathological study. J Pak Med Assoc. 1991;41: 86-88.

8. Kutty MK, Anim JT, Sowayan S. Histiocytic necrotizing lymphadenitis (Kikuchi-Fujimoto disease) in Saudi Arabia. Trop Geogr Med. 1991;43:68-75.

9. Suseelan AV, Augusty TS, Harilal KR. Necrotizing lymphadenitis: an analysis of 17 cases. Indian $J$ Pathol Microbiol. 1984; 27: 331-334.

10. Tsang WYW, Chan JKC, Ng CS. Kikuchi's lymphadenitis: amorphologic analysis of 75 cases with special reference tounusual features. Am J Surg Pathol. 1994; 18: 219-231.

11. Kuo T. Kikuchi's disease (histiocytic necrotizing lymphadenitis): a clinicopathologic study of 79 cases with ananalysis of histologic subtypes, immunohistology, and DNA ploidy. Am J Surg Pathol. 1995; 19: 798-809.

12. Case Records of the Massachusetts General Hospital (Case 5-1997). N Engl J Med. 1997;336:492-499.

13. Pileri S, Kikuchi M, Helbron D, et al. Histiocytic necrotizing lymphadenitis without granulocytic infiltration. Virchows Arch A Pathol Anat Histopathol. 1982; 395: 257-271. 
14. AKM Mosharraf-Hossain,Pran Gopal Datta, AS Ahmed Amin, M Jalal Uddin, Kikuchi-Fujimoto Disease presenting with fever,lymphadenopathy and dysphagia. J Pak Med Assoc. 2008. Vol. 58, No. $11: 647-649$

15. Yen A, Fearneyhough P, Raimer SS, et al. EBVassociated Kikuchi's histiocytic necrotizing lymphadenitis with cutaneous manifestations. $J$ Am Acad Dermatol. 1997;36:342-346.

16. Dorfman RF, Berry GJ. Kikuchi's histiocytic necrotizing lymphadenitis: an analysis of 108 cases with emphasis on differential diagnosis. Semin Diagn Pathol. 1988;5:329-345.

17. Lin HC, Su CY, Huang CC, et al. Kikuchi's disease: a review and analysis of 61 cases. Otolaryngol Head Neck Surg. 2003;128:650-653.

18. Feller AC, Lennert K, Stein $H$, et al. Immunohistochemistry and etiology of histiocytic necrotizing lymphadenitis: report of three instructive cases. Histopathology. 1983;7:825-839.

19. Heikens J, Tel W, van de Stadt J, et al. Kikuchi's lymphadenitis: report of a Yersinia enterocoliticaassociated case and an overview of aetiology and clinical outcome. Neth J Med. 1992;41:222-228.

20. Cho KJ, Lee SS, Khang SK. Histiocytic necrotizing lymphadenitis: a clinicopathologic study of 45 cases with in situ hybridization for Epstein-Barr virus and hepatitis B virus. J Korean Med Sci. 1996;11:409414.

21. Chiu CF, Chow KC, Lin TY, et al. Virus infection in patients with histiocytic necrotizing lymphadenitis in Taiwan:detection of Epstein-Barr virus, type I human T-cell lymphotropic virus, and parvovirus B19. Am J Clin Pathol. 2000;113:774-781.

22. Hollingsworth HC, Peiper SC, Weiss LM, et al. An investigation of the viral pathogenesis of KikuchiFujimoto disease: lack of evidence for Epstein-Barr virus or human herpesvirus type 6 as the causative agents. Arch Pathol Lab Med. 1994;118:134-140.

23. Georges TI, Jones CD, Zehnder JL, et al. Lack of human herpesvirus 8 and Epstein-Barr virus in Kikuchi's histiocytic necrotizing lymphadenitis. Hum Pathol. 2003;34:130-135.

24. Sumiyoshi Y, Kikuchi M, Minematu T, et al. Analysis of herpesvirus genomes in Kikuchi's disease. Virchows Arch. 1994;424:437-440.

25. Huh J, Chi HS, Kim SS, et al. A study of the viral etiology of histiocytic necrotizing lymphadenitis (Kikuchi-Fujimoto disease). J Korean Med Sci. 1998;13:27-30.

26. Sumiyoshi Y, Kikuchi M, Ohshima K, et al. Human herpesvirus- 6 genomes in histiocytic necrotizing lymphadenitis (Kikuchi's disease) and other forms of lymphadenitis. Am J Clin Pathol. 1993;99:609614.
27. Imamura $M$, Ueno $H$, Matsuura A, et al. An ultrastructural study of subacute necrotizing lymphadenitis. Am J Pathol. 1982;107:292-299.

28. Tanaka T, Ohmori M, Yasunaga S, et al. DNA typing of HLA class II genes (HLA-DR, -DQ and -DP) in Japanese patients with histiocytic necrotizing lymphadenitis (Kikuchi's disease). Tissue Antigens. 1999;54:246-253.

29. Asano S, Akaike Y, Muramatsu T, et al. Necrotizing lymphadenitis: a clinicopathological and immunohistochemical study of four familial cases and five recurrent cases. Virchows Arch A Pathol Anat Histopathol. 1991;418:215-223.

30. Felgar RE, Furth EE, Wasik MA, et al. Histiocytic necrotizing lymphadenitis (Kikuchi's disease): in situ end-labeling, immunohistochemical, and serologic evidence supporting cytotoxic lymphocyte-mediated apoptotic cell death. Mod Pathol. 1997;10:231-241.

31. Ohshima K, Shimazaki K, Suzumiya J, et al. Apoptosis of cytotoxic T-cells in histiocytic necrotizing lymphadenitis. Virchows Arch. 1998;433:131-134.

32. Ohshima K, Shimazaki K, Kume T, et al. Perforin and Fas pathways of cytotoxic T-cells in histiocytic necrotizing lymphadenitis. Histopathology. 1998;33:471-478.

33. Galibert L, Maliszewski CR, Vandenabeele S. Plasmacytoid monocytes/T cells: a dendritic cell lineage? Semin Immunol. 2001;13:283-289.

34. Comeau MR, Van der Vuurst de Vries AR, Maliszewski CR, et al. CD123bright plasmacytoid predendritic cells: progenitors undergoing cell fate conversion? J Immunol. 2002;169:75-83.

35. Abe Y, Ohshima K, Nakashima M, et al. Expression of apoptosis-associated protein RCAS1 in macrophages of histiocytic necrotizing lymphadenitis. Int $J$ Hematol. 2003;77:359-363.

36. Kato K, Ohshima K, Anzai K, et al. Elevated serumsoluble Fas ligand in histiocytic necrotizing lymphadenitis. Int $J$ Hematol. 2001;73:84-86.

37. Kuo TT. Kikuchi's disease (histiocytic necrotizing lymphadenitis): a clinicopathologic study of 79 cases with an analysis of histologic subtypes, immunohistology, and DNA ploidy. Am J Surg Pathol 1995; 19:798-809.

38. Bosch X, Guilabert A, Miquel R, Campo E. Enigmatic Kikuchi- Fujimoto disease: a comprehensive review. Am J clin Pathol .2004;122:141-52.

39. Osborn M, Aqel N, Levine TS. The fine needle aspiration appearances of Kikuchi's lymphadenitis. Cytopathology 2009;20:36-43.

40. Kampitak T. Fatal Kikuchi-Fujimoto disease associated with SLE and hemophagocytic syndrome: a case report. Clin Rheumatol. Aug 2008;27(8):10735 . 
41. Singh YP, Agarwal V, Krishnani N, Misra R. Enthesitis-related arthritis in Kikuchi-Fujimoto disease. Mod Rheumatol. May 10 2008; epub ahead of print.

42. Lee S, Yoo JH, Lee SW. Kikuchi Disease: Differentiation from Tuberculous Lymphadenitis Based on Patterns of Nodal Necrosis on CT. AJNR Am J Neuroradiol. Dec 152011.

43. Hutchinson CB, Wang E. Kikuchi-Fujimoto disease. Arch Pathol Lab Med. Feb 2010;134(2):289-93.

44. Sopeña B, Rivera A, Vázquez-Triñanes C, Fluiters E, González-Carreró J, Del Pozo M, et al. Autoimmune Manifestations of Kikuchi Disease. Semin Arthritis Rheum. Dec 20 2011;

45. Atwater AR, Longley BJ, Aughenbaugh WD. Kikuchi's disease: case report and systematic review of cutaneous and histopathologic presentations. $J$ Am Acad Dermatol. Jul 2008;59(1):130-6.

46. Yasukawa K, Matsumura T, Sato-Matsumura KC, et al. Kikuchi's disease and the skin: case report and review of the literature. $\mathrm{Br} J$ Dermatol. Apr 2001;144(4):885-9.

47. Sato Y, Kuno H, Oizumi K. Histiocytic necrotizing lymphadenitis (Kikuchi's disease) with aseptic meningitis. J Neurol Sci. Mar 1 1999;163(2):187-91.

48. Kapadia Y, Robinson BA, Angus HB. Kikuchi's disease presenting as fever of unknown origin [letter]. Lancet.1989;2:1519-1520.

49. el-Ramahi KM, Karrar A, Ah MA. Kikuchi disease and its association with systemic lupus erythematosus. Lupus.1994;3:409-411.

50. Martinez-Vazquez C, Hughes G, Bordon J, et al. Histiocytic necrotizing lymphadenitis, KikuchiFujimoto's disease,associated with systemic lupus erythematosus. QJM. 1997;90:531-533.

51. Litwin MD, Kirkham B, Henderson DRF, et al. Histiocytic necrotising lymphadenitis in systemic lupus erythematosus. Ann Rheum Dis. 1992;151:805807.

52. Pappaioannou G, Speletas M, Kaloutsi V, et al. Histiocytic necrotizing lymphadenitis (KikuchiFujimoto disease) associated with antiphospholipid syndrome: case report and literature review. Ann Hematol. 2002;81:732-735.

53. Wilkinson CE, Nichol F. Kikuchi-Fujimoto disease associated with polymyositis. Rheumatology (Oxford). 2000;39:1302-1304.

54. Ramanan AV, Wynn RF, Kelsey A, et al. Systemic juvenile idiopathic arthritis, Kikuchi's disease and haemophagocytic lymphohistiocytosis: is there a link? case report and review of the literature. Rheumatology (Oxford). 2003;42:596-598.

55. Taguri AH, McIlwaine GG. Bilateral panuveitis: a possibleassociation with Kikuchi-Fujimoto disease. Am J Ophthalmol.2001;132:419-421.
56. Graham LE. Kikuchi-Fujimoto disease and peripheral arthritis: a first [letter]! Ann Rheum Dis. $2002 ; 61: 475$.

57. Belagavi CS, Kubeyinje EP. Recurrent cutaneous necrotizing vasculitis in a patient with Kikuchi's disease: case report. East Afr Med J. 1997;74:747748.

58. Wong CY, Law GT, Shum TT, et al. Pulmonary haemorrhage in a patient with Kikuchi disease. Monaldi Arch Chest Dis.2001;56:118-120.

59. Pasquinucci S, Dionisi PM, Cavinato F, et al. Kikuchi's disease in a patient infected with AIDS [letter]. AIDS. 1991;5:235.

60. Kelly J, Kelleher K, Khan MK. A case of haemophagocytic syndrome and Kikuchi-Fujimoto disease occurring concurrently in a 17 -year-old female. Int $J$ Clin Pract. 2000;54:547-549.

61. Mahadeva U, Allport $\mathrm{T}$, Bain $\mathrm{B}$, et al. Haemophagocytic syndrome and histiocytic necrotising lymphadenitis (Kikuchi's disease). J Clin Pathol. 2000;53:636-638.

62. Laccourreye O, Kugelstadt P, Hugues FC, et al. Intraparotid subacute necrotizing histiocytic lymphadenitis (Kikuchi- Fujimoto's disease). J Otolaryngol. 1997;26:64-66.

63. Keogh MA, Williamson RM, Denaro CP. Kikuchi's disease associated with parotidomegaly, thyroiditis and a rash in a young man [letter]. Aust $N Z \mathrm{~J} \mathrm{Med}$. 2000;30:633-634. Am J Clin Pathol 2004;122:141152

64. Radhi JM, Skinnider L, McFadden A. Kikuchi's lymphadenitis and carcinoma of the stomach. J Clin Pathol.1997;50:530-531.

65. Yoshino T, Mannami T, Ichimura K, et al. Two cases of histiocytic necrotizing lymphadenitis (KikuchiFujimoto'sdisease) following diffuse large B-cell lymphoma. Hum Pathol. 2000;31:1328-1331.

66. Kaur S, Thami GP, Mohan H, et al. Kikuchi disease with facial rash and erythema multiforme. Pediatr Dermatol.2001;18:403-405.

67. Spies J, Foucar K, Thompson C, et al. The histopathology of cutaneous lesions of Kikuchi's disease: a report of 5 cases. Am J Surg Pathol. 1999;23:1040-1047.

68. Sumiyoshi Y, Kikuchi M, Ohshima K, et al. A case of histiocytic necrotizing lymphadenitis with bone marrow and skin involvement. Virchows Arch A Pathol Anat Histopathol. 1992;420:275-279.

69. Youk JH, Kim EK, Ko KH, Kim MJ. Sonographic features of axillary lymphadenopathy caused by Kikuchi disease. J Ultrasound Med. Jun 2008;27(6):847-53.

70. Kwon SY, Kim TK, Kim YS, et al. CT findings in Kikuchi disease: analysis of 96 cases. AJNR Am J Neuroradiol. Jun-Jul 2004;25(6):1099-102. 
71. Tong TR, Chan OW, Lee KC. Diagnosing Kikuchi disease on fine needle aspiration biopsy: a retrospective study of 44 cases diagnosed by cytology and 8 by histopathology. Acta Cytol. Nov-Dec 2001; 45(6): 953-7.

72. Viguer JM, Jiménez-Heffernan JA, Pérez P, et al. Fine-needle aspiration cytology of Kikuchi's lymphadenitis: a report of ten cases. Diagn Cytopathol. Oct 2001;25(4):220-4.

73. Singh YP, Agarwal V, Krishnani N, Misra R. Enthesitis-related arthritis in Kikuchi-Fujimoto disease. Mod Rheumatol. May 10 2008; epub ahead of print.
74. Jang YJ, Park KH, Seok HJ. Management of Kikuchi's disease using glucocorticoid. J Laryngol Otol. Sep 2000;114(9):709-11.

75. M. Noursadeghi, N. Aquel, P. Gibaon, G. Pasvol. Successful treatment of severe Kikuchi's disease with intravenous immunoglobulin. Rheumatology. 2006; 45: $237-238$

76. Kim YM, Lee YJ, Nam SO, Park SE, Kim JY, Lee EY. Hemophagocytic syndrome associated with Kikuchi's disease.J Korean Med Sci 2003;18: 5924.

77. Chen JS, Chang KC, Cheng CN, Tsai WH, Su IJ. Childhood hemophagocytic syndrome associated with Kikuchi's disease. Haematologica 2000;85:9981000 